\title{
SISTEMAS NANOESTRUTURADOS CONTENDO ÓLEO DE LINHAÇA: desenvolvimento tecnológico e caracterização físico-química de nanoemulsões e nanocápsulas poliméricas
}

\author{
NANOSTRUTURED SYSTEMS CONTAINING LINSEED OIL: \\ technological development and physicochemical characterization \\ of nanoemulsions and polymeric nanocapsules
}

\section{Cristina Rigon', Laura Minussi Giuliani', Mônica Fabiele Lorensi Stangarlin², Juliane Mattiazzi², Felipe Pereira Gomes ${ }^{1}$, Natháli Schopf Pegoraro², Letícia Cruz ${ }^{3}$, Luana Mota Ferreira²}

\section{RESUMO}

Objetivo: O óleo de linhaça apresenta diversas propriedades terapêuticas, principalmente devido à presença de ácidos poli-insaturados em sua composição. Para melhor eficácia e/ou aumento da estabilidade de óleos vegetais farmacologicamente ativos, sistemas nanoestruturados são desenvolvidos para veiculação deste tipo de substância. Este estudo objetivou desenvolver e caracterizar sistemas nanoestruturados contendo óleo de linhaça. Foram preparadas nanocápsulas poliméricas e nanoemulsões, avaliando-se duas concentrações de óleo (1,5\% e 3,0\%) em ambas as formulações e dois polímeros, Poli(E-caprolactona) e Eudragit ${ }^{\circledR} \mathrm{RL} 100$, no desenvolvimento das nanocápsulas. Ambas as formulações apresentaram características adequadas para sistemas coloidais. Em relação às nanoemulsões, houve diferença significativa das características entre as quantidades de óleo, sendo que 1,5\% de óleo de linhaça foi a concentração mais satisfatória. Em relação à presença dos polímeros, houve variação apenas dos valores de potencial zeta e pH. Concluindo, as formulações foram consideradas adequadas para o encapsulamento do óleo de linhaça, sendo carreadores promissores para outras substâncias ativas.

Descritores: Óleos Vegetais; Óleo de Linhaça; Nanoemulsões: Nanocápsulas Poliméricas.
${ }^{1}$ Graduanda(o) em Farmácia na Universidade Federal de Santa Maria (UFSM), Santa Maria, RS, Brasil.

2 Mestre em Ciências Farmacêuticas pela Universidade Federal de Santa Maria (UFSM), Santa Maria, RS, Brasil.

${ }^{3}$ Doutora em Ciências Farmacêuticas pela Universidade Federal do Rio Grande do Sul (UFRGS), Porto Alegre, RS, Brasil.

\begin{abstract}
Objective: Linseed oil has several therapeutic properties, mainly due to the presence of polyunsaturated acids in its composition. For best efficiency and/or increasing the stability of vegetable oils pharmacologically active, nanoestructured systems are developed to vehicle this type of substance. This study aimed to develop and characterize nanostructured systems containing linseed oil. Polymeric nanocapsules, and nanoemulsions were prepared, evaluating two oil concentrations $(1.5 \%$ and $3.0 \%)$ in both formulations and two polymers, Poli( $\left(\right.$-caprolactona) e Eudragit ${ }^{\circledR}$ RL100, of the nanocapsules development. Both formulations showed appropriate properties for colloidal systems. Regarding the nanoemulsions, there was a significant difference in characteristics between the amounts of oil, while $1.5 \%$ linseed oil was the most satisfying concentration. About presence of polymers, there was variation only of zeta potential and $\mathrm{pH}$ values. In conclusion, the formulations were considered suitable for the encapsulation of the linseed oil, being promising carriers for other active substances.
\end{abstract}




\section{Introdução}

Óleos vegetais, em sua grande maioria, apresentam inúmeras propriedades nutricionais benéficas ao organismo humano. A linhaça (Linumu sitatissimum L.) é uma planta cultivada por séculos em países do Mediterrâneo e representa a semente do linho. É através dela que após os processamentos pode-se obter o óleo de linhaça $(\mathrm{OL})^{1}$. A semente de linhaça apresenta componentes biologicamente ativos importantes como ácido a-linolênico (ALA), diglicosídeo secoisolariciresinol e fibras dietéticas, o que vem gerando interesse de estudos em diferentes áreas, procurando associar estes princípios ativos aos efeitos benéficos para a saúde ${ }^{2}$. A presença destas substâncias conferem ao OL algumas atividades farmacológicas como potencial anti-inflamatório e redução de tumores, especialmente os hormônios-sensíveis ${ }^{2,3}$.

O OL possui insaturações em sua estrutura e apresenta quantidade abundante de ácido a-linolênico ${ }^{4}$. Ácidos graxos que apresentam múltiplas insaturações abrangem os tipos ômega-3 e ômega-6, dependendo da localização da dupla ligação, e pela proximidade do grupamento metil da extremidade da cadeia ${ }^{5}$. Esses ácidos graxos são denominados essenciais, e devem fazer parte da dieta humana, já que o organismo é incapaz de sintetizá-los. Além disso, os ácidos graxos poli-insaturados estão associados à prevenção de doenças cardiovasculares e inflamatórias ${ }^{6}$.

Devido às potencialidades do OL, torna-se relevante sua incorporação em formulações que favoreçam sua absorção no organismo e protejam-no contra eventuais fenômenos de degradação. Nesse sentido, por ser um óleo vegetal com propriedades nutricionais e energéticas tão relevantes à saúde humana, é importante que seus constituintes sejam protegidos de processos como a oxidação lipídica, uma vez que trata-se de um óleo poli-insaturado ${ }^{7}$. A fotooxidação e a oxidação enzimática interferem diretamente nas características organolépticas, no valor nutricional do óleo e até mesmo, podem gerar compostos nocivos à saúde ${ }^{7}$.

0 crescente interesse no desenvolvimento de nanoestruturas coloca esta área em destaque, principalmente devido às vantagens que estes sistemas coloidais apresentam frente às formulações convencionais. Os sistemas nanoestruturados são capazes de controlar a liberação dos ativos/fármacos incorporados, proteger tais substâncias frente à degradação química, física e enzimática, e melhorar a absorção no trato gastrointestinal| ${ }^{8,9}$. A nanotecnologia, na área da indústria alimentar, tem se mostrado promissora no que diz respeito ao encapsulamento de aromas, sabores e substâncias nutracêuticas ${ }^{10-12}$. Além disso, os nanossistemas influenciam aspectos como a liberação de ingredientes funcionais presentes no alimento até um sítio de ação específico e ainda contribuem para mantê-los em seu estado ativo durante o seu processamento. Ainda, cabe ressaltar também que a nanotecnologia pode estar presente nas etapas póscolheita e durante o processamento ${ }^{13}$. Dentre os sistemas nanotecnológicos mais estudados é relevante destacar as nanoemulsões e as nanopartículas poliméricas ${ }^{14,15}$.

Por definição, nanopartículas poliméricas são consideradas partículas sólidas coloidais, e dividem-se em nanoesferas e nanocápsulas ${ }^{16}$, de acordo com a estrutura e composição. As nanocápsulas são constituídas por um invólucro polimérico em torno de um núcleo oleoso, sendo que o princípio ativo pode estar dissolvido no núcleo e/ou adsorvido ao polímero. Já as nanoesferas são matrizes poliméricas, nas quais o princípio ativo pode ficar retido ou adsorvido no polímero ${ }^{14,17}$. As nanoemulsões correspondem a gotículas de óleo de tamanho nanométrico dispersas em uma fase aquosa ${ }^{18}$.

Tendo em vista que os componentes das formulações influenciam nas características, a escolha do tipo e concentração deve ser feita de forma criteriosa. No caso das nanocápsulas, a escolha dos polímeros é muito importante, uma vez que além de existir uma variedade dos mesmos, eles podem influenciar no perfil de liberação, tamanho de partícula e potencial zeta do sistema nanoestruturado ${ }^{19}$. O polímero deve ser biocompatível, podendo ou não ser biodegradável, de acordo com a via de administração que se deseja. Dentre estes, pode-se citar a poli(£-caprolactona) (PCL), um poliéster biocompatível e biodegradável, e o Eudragit ${ }^{\circledR}$ RL100 (EUD RL100), um derivado do ácido metacrílico de característica catiônica.

Considerando o que foi exposto, o objetivo deste trabalho foi delinear uma formulação de base nanotecnológica para veicular o OL, bem como avaliar a influência do tipo e concentração dos componentes escolhidos no desenvolvimento das nanocápsulas e nanoemulsões através da caracterização físico-química das mesmas. 


\section{Metodologia}

\section{Matérias-primas}

O óleo de linhaça foi obtido da Campestre, a Poli(E-caprolactona) e o monooleato de sorbitano (Span $\left.{ }^{\circledR} 80\right)$ da Sigma Aldrich. Eudragit ${ }^{\circledR}$ RL100 foi fornecido pela Degussa, o polissorbato 80 (Tween ${ }^{\circledR} 80$ ) pela Delaware e acetona foi obtida da Nuclear.

\section{Teste de inchamento/dissolução dos filmes poliméricos de PCL e EUD RL100}

Filmes de PCL foram obtidos por prensagem hidráulica e de EUD RL100 obtidos por dispersão em acetona. Após, foram imersos completamente em $2 \mathrm{~mL}$ de OL. Os filmes foram cuidadosamente retirados do contato com o OL, secos com papel e pesados em balança analítica calibrada após 1, 3, 5, 7, 11, 28 e 60 dias de imersão. 0 ensaio foi realizado em triplicata ${ }^{20-21}$.

\section{Perfil Espectrofotométrico do óleo de linhaça na região ultravioleta}

Os espectros de absorção do OL foram obtidos através da varredura de uma solução etanólica contendo o óleo a uma concentração de 1,5 mg/mL em espectrofotômetro na faixa de 190 - $400 \mathrm{~nm}$, abrangendo os espectros de absorção das radiações UVC, UVB e UVA (Shimadzu, modelo UV-1800).

\section{Preparação dos sistemas nanoestruturados}

Foram preparadas nanoemulsões (NE) e suspensões de nanocápsulas (NC) com os polímeros PCL ou EUD RL100, contendo OL. As suspensões de nanocápsulas foram preparadas através do método da deposição interfacial do polímero pré-formado ${ }^{22}$. Uma fase orgânica, composta de acetona, Span ${ }^{\circledR} 80$, PCL ou EUD RL100 e óleo de linhaça foi mantida sob agitação magnética por 60 minutos a $40^{\circ} \mathrm{C} \mathrm{e}$, em seguida, vertida em uma fase aquosa contendo polisorbato 80. A mistura permaneceu sob agitação magnética por 10 minutos e foi levada ao evaporador rotatório para remoção do solvente orgânico e ajuste do volume final para $10 \mathrm{~mL}$ de formulação. As nanoemulsões foram preparadas através do método da emulsificação espontânea ${ }^{23}$, como descrito para nanocápsulas, excluindo-se o polímero da formulação. A composição quali e quantitativa das formulações, bem como suas respectivas nomenclaturas, encontram-se na tabela 1.

Tabela 1 - Composição das nanoestruturas

\begin{tabular}{|c|c|c|c|c|c|c|}
\hline & NE-A & NE-B & NC-PCL-A & NC-PCL-B & NC-EUD-A & NC-EUD-B \\
\hline \multicolumn{7}{|l|}{ Fase aquosa } \\
\hline Tween $^{\circledast} 80$ (g) & 0,077 & 0,077 & 0,077 & 0,077 & 0,077 & 0,077 \\
\hline Água destilada $(\mathrm{mL})$ & 53 & 53 & 53 & 53 & 53 & 53 \\
\hline \multicolumn{7}{|l|}{ Fase orgânica } \\
\hline EUD (g) & - & - & - & - & 0,100 & 0,100 \\
\hline $\mathrm{PCL}(\mathrm{g})$ & - & - & 0,100 & 0,100 & - & - \\
\hline $\operatorname{Span}^{\circledast} 80(\mathrm{~g})$ & 0,077 & 0,077 & 0,077 & 0,077 & 0,077 & 0,077 \\
\hline Acetona $(\mathrm{mL})$ & 27 & 27 & 27 & 27 & 27 & 27 \\
\hline OL (g) & 0,3 & 0,15 & 0,3 & 0,15 & 0,3 & 0,15 \\
\hline
\end{tabular}

EUD: Eudragit RL100; PCL: Poli(ع-caprolactona), OL: Óleo de linhaça. 


\section{Caracterização Físico-Química}

As técnicas de caracterização físico-química empregadas neste estudo são aquelas consideradas essenciais para descrever um sistema nanoestruturado ${ }^{14}$. Tais metodologias já vem sendo utilizadas pelo nosso grupo de pesquisa para avaliar as caraterísticas dos sistemas nanométricos ${ }^{20,24,25,26}$.

\section{Determinação do pH}

$\mathrm{O} \mathrm{pH}$ foi determinado diretamente nas amostras mediante a utilização de potenciômetro (HANNA, modelo pH 21), calibrado com soluções tampão de pH 4,0 e 7,0, sem diluição.

\section{Determinação do tamanho, índice de polidispersão e potencial zeta}

A avaliação do tamanho das partículas e das gotículas e 0 índice de polidispersão (IPD) das mesmas foi realizada por espectroscopia de correlação de fótons $\left(25^{\circ} \mathrm{C}\right)$, após diluição adequada de uma alíquota dos sistemas nanoestruturados em água (equipamento Zetasizer Nanoseries Malvern Instruments, modelo ZEN 3600). Por sua vez, os valores de potencial zeta foram determinados por microeletroforese, após diluição das amostras em $\mathrm{NaCl}(10 \mathrm{mM})$ no mesmo equipamento ${ }^{20-26}$.

\section{Análise estatística}

As formulações foram preparadas e analisadas em triplicata e os resultados foram analisados por Teste $t$ (para comparação do tempo inicial e final de teste de inchamento/dissolução dos filmes poliméricos) e análise de variância (ANOVA) de uma via, seguida de post-hoc Tukey (para as análises de caracterização físico-química), utilizando o programa GraphPad Prism, versão 6. Valores de $p<0,05$ foram considerados estatisticamente significativos.

\section{Resultados}

Após a preparação, as nanoemulsões e as suspensões de nanocápsulas apresentaram aspecto branco-leitoso e efeito Tyndall, o qual é resultado do movimento browniano de sistemas coloidais. Não foram observados precipitados ou quaisquer outras alterações nas formulações.

Os filmes poliméricos de EUD RL100 e PCL foram imersos no OL com o intuito de verificar alterações ou interações ao longo de 60 dias. Após o período de imersão, nenhuma alteração significativa foi observada (Teste $t, p>0,05$ ). Os resultados são demonstrados na figura 1.

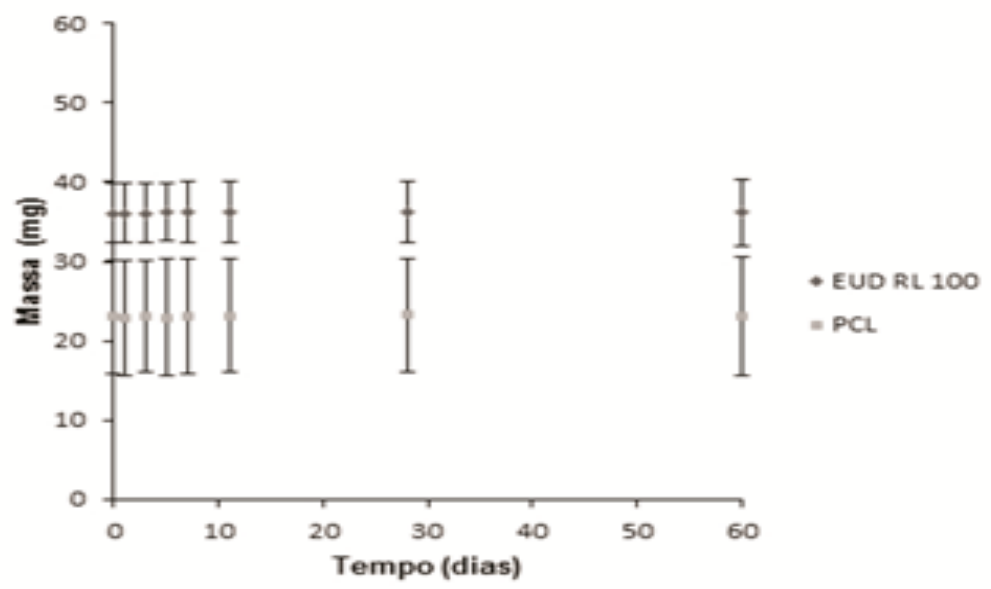

Figura 1 - Massa dos filmes de Eudragit ${ }^{\oplus}$ RL100 e de Poli ( $\varepsilon$-caprolactona) no decorrer de 60 dias de imersão em óleo de linhaça. 
Outra etapa de pré-formulação a ser determinada é o perfil de absorção espectrofotométrica da substância em estudo. 0 espectro de absorção do óleo de linhaça em solução etanólica pode ser observado na figura 2, na faixa de comprimento de onda entre 190 a $400 \mathrm{~nm}$, a partir do qual nota-se que a maior absorção encontra-se entre 190 a $290 \mathrm{~nm}$.

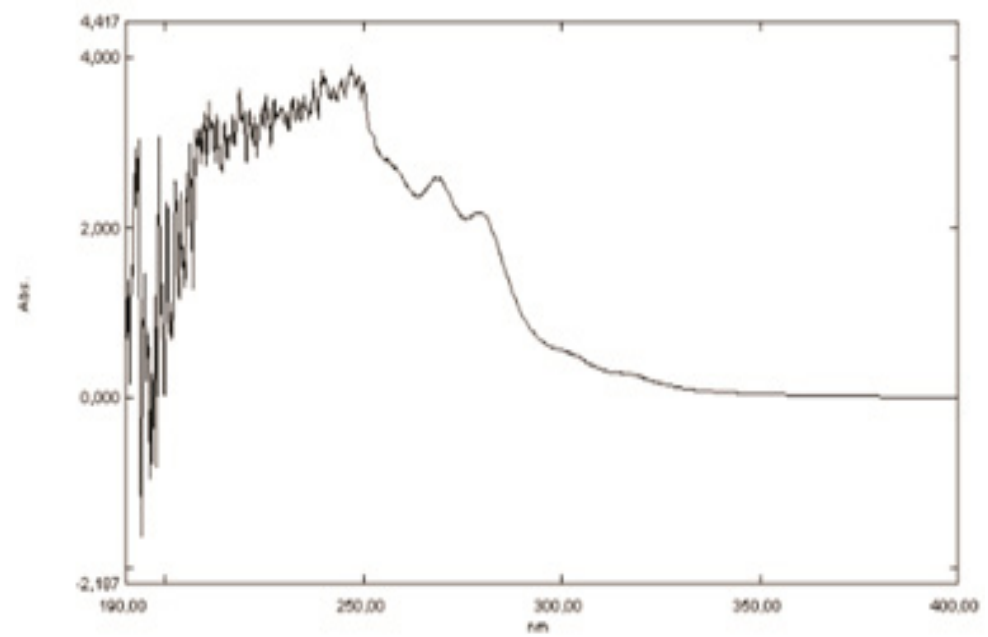

Figura 2 - Perfil de absorção espectrofotométrica do óleo de linhaça.

Após a realização destes estudos preliminares, foram avaliadas as características físico-químicas dos nanossistemas desenvolvidos, conforme mostra a tabela 2. 0 diâmetro das partículas/gotículas permaneceu em escala nanométrica, variando entre $295 \pm 32$ a $191 \pm 6 \mathrm{~nm}$. Por sua vez, o índice de polidispersão esteve em torno de 0,2. Os valores de potencial zeta para as formulações contendo PCL foram negativos, assim como para as nanoemulsões, enquanto que as nanocápsulas compostas por EUD RL100 apresentaram valores positivos. Os resultados do pH das formulações variaram de 5,46 \pm 0,33 a 6,86 $\pm 0,12$, sendo que as suspensões de nanocápsulas de EUD RL100 apresentaram valores na faixa ácida.

Tabela 2 - Caracterização físico-química das formulações

\begin{tabular}{c|c|c|c|c}
\hline Formulação & $\begin{array}{c}\text { Tamanho } \\
(\mathbf{n m})\end{array}$ & $\begin{array}{c}\text { Índice de } \\
\text { polidispersão }\end{array}$ & $\begin{array}{c}\text { Potencial } \\
\text { Zeta (mV) }\end{array}$ & $\mathbf{p H}$ \\
\hline NE-A & $263 \pm 28$ & $0,28 \pm 0,06^{\mathrm{a}}$ & $-18,68 \pm 3,19$ & $6,73 \pm 0,15$ \\
\hline NE-B & $191 \pm 6$ & $0,12 \pm 0,03$ & $-15,07 \pm 3,76$ & $6,41 \pm 0,26$ \\
\hline NC-PCL-A & $295 \pm 32$ & $0,19 \pm 0,03$ & $-17,66 \pm 7,23$ & $6,86 \pm 0,12$ \\
\hline NC-PLC-B & $260 \pm 39$ & $0,20 \pm 0,05$ & $-20,90 \pm 1,40$ & $6,85 \pm 0,01$ \\
\hline NC-EUD-A & $236 \pm 23$ & $0,25 \pm 0,05$ & $15,38 \pm 1,54$ & $5,63 \pm 1,08^{b}$ \\
\hline NC-EUD-B & $193 \pm 9$ & $0,16 \pm 0,02$ & $16,67 \pm 2,07$ & $5,46 \pm 0,33^{b}$ \\
\hline
\end{tabular}

$a$ = diferença significativa entre NE-A e NE-B; $b$ = diferença estatística entre NC-EUD-A e NC-EUD-B com as demais formulações.

\section{Discussão}

A combinação dos constituintes de uma formulação e também o método de preparação interferem diretamente em propriedades importantes das nanoestruturas e, consequentemente, na estabilidade ${ }^{14}$. Este trabalho demonstra 0 desenvolvimento de formulações de base nanotecnológica bem como a avaliação da influência do tipo e da quantidade dos componentes nas características físico-químicas dos sistemas contendo OL. 
No desenvolvimento de um sistema nanoestruturado como as nanocápsulas é importante elucidar as possíveis interações existentes entre o polímero e o óleo de escolha em termos de inchamento e dissolução. Óleos podem compor o núcleo oleoso de nanocápsulas desde que o polímero empregado não sofra dissolução ou inchamento quando em contato com o óleo ${ }^{27}$. A partir dos resultados pode-se verificar que os polímeros são adequados para a preparação das nanocápsulas de óleo de linhaça, pois não houve alteração significativa no peso dos filmes poliméricos, indicando que não ocorreu nenhuma interação entre as matérias-primas (Teste $t, p>0,05$ ).

Como outra etapa de pré-formulação o perfil de absorção espectrofotométrica do OL foi avaliado. Este teste é simples, fácil e pode ser útil para prever fenômenos de instabilidade pela absorção de radiação. Com base na análise do espectro, observou-se uma maior absorção da radiação ultravioleta na faixa de comprimento de onda de 190 a 290 nm. Esta faixa de absorção compreende a região UVC e, assim, sugere-se que o OL seja capaz de proteger substâncias fotoinstáveis associadas às nanoestruturas. Em alguns estudos que empregam óleos vegetais como componente oleoso de sistemas nanoestruturados é possível observar aumento na estabilidade de ativos como, por exemplo, dipropionato de beclometasona, clotrimazol e cetoprofeno $0^{20-21-25}$.

Após os estudos de pré-formulação, as nanoestruturas foram preparadas por métodos que já estão bem descritos na literatura e que são considerados fáceis e rápidos para a obtenção das partículas/gotículas coloidais. As nanoemulsões contendo óleo de linhaça foram preparadas através do método de emulsificação espontânea nas concentrações de óleo de 3,0\% (NE-A) e de 1,5\% (NE-B). As formulações NE-B apresentaram resultados de tamanho de gotícula menores que NE-A, mas não foi observada diferença significativa entre as formulações (ANOVA, 1 via, $p<0,05$ ). Nanoemulsões geralmente apresentam-se na faixa de tamanho de 100 a $600 \mathrm{~nm}$, sendo relevante mencionar que o tamanho reduzido das nanoemulsões garante além de estabilidade cinética, formulações mais translúcidas e de menor viscosidade ${ }^{23-28}$. Tamanho de gotícula semelhante ao encontrado nas NE-B também foi observado em outro estudo, no qual incorporaram o óleo de linhaça em nanoemulsões que apresentaram diâmetro de gotícula de 176,6 \pm 18,2 nm²9.

Valores elevados de índice de polidispersão caracterizam um sistema com heterogeneidade no tamanho das partículas/gotículas. Sendo assim as nanoemulsões contendo 1,5\% de óleo apresentaram uma distribuição de tamanho mais homogênea em relação a NE-A, uma vez que valores de índice de polidispersão em torno de 0,2 mostram uma estreita faixa de distribuição de diâmetro30. Houve uma redução significativa no índice de polidispersão pela diminuição da concentração do óleo (ANOVA, 1 via, $p<0,05$ ). Alguns autores relatam que com o aumento da viscosidade da fase oleosa, a difusão do solvente é mais lenta durante a preparação e, consequentemente, ocorre a formação de gotículas maiores e menos homogêneas ${ }^{23,31}$.

Quanto ao estudo do potencial zeta, este, em módulo, quanto mais distante de zero indica boa estabilidade físicoquímica ao evitar a agregação das suspensões ${ }^{14}$. Assim, para ambas as concentrações de óleo estudadas, os valores foram negativos. Através do monitoramento do $\mathrm{pH}$ das suspensões ao longo do tempo também é possível predizer a estabilidade das formulações e explicar fenômenos de instabilidade que podem estar relacionados, por exemplo, a processos de degradação dos constituintes ou compatibilidade com a via de administração ${ }^{14}$. Conforme observou-se nas análises, o pH das formulações manteve-se na faixa ácida, o que pode ser explicado pela presença dos ácidos graxos existentes no OL, e, no caso de administração oral da formulação, os valores encontram-se em uma faixa de pH bastante semelhante com a do intestino delgado e, mais especificamente, no duodeno, a qual varia entre 6,5 a 7,632. Ademais, não houve diferença significativa entre as formulações para esses parâmetros (ANOVA, 1 via, $p>0,05$ ).

Com relação às NC de PCL, o tamanho das partículas manteve-se na faixa de $260 \pm 39$ nm para as de menor concentração de OL, enquanto que as formulações contendo $3,0 \%$ de OL apresentaram valores mais elevados ( $295 \pm 32 \mathrm{~nm}$ ). Na literatura são encontrados trabalhos que mostram a associação de diferentes óleos a nanoestruturas de PCL como polímero nos quais também foram avaliados o tamanho das partículas em suspensão20,33,34. Em outro trabalho no qual foram desenvolvidas suspensões de nanocápsulas também pelo método de deposição interfacial do polímero PCL, observou-se partículas com tamanhos menores que $270 \mathrm{~nm}$, valores estes bastante semelhantes aos encontrados neste estudo para a formulação NC-PCL-B ${ }^{30}$.

O índice de polidispersão mostrou que as nanocápsulas de PCL são homogêneas quanto ao tamanho, uma vez que os valores ficaram abaixo ou igual a 0,2. Quanto ao potencial zeta, apresentou-se negativo, o qual pode ser justificado pela presença de grupo carboxílicos terminais do polímero empregado e pelos ácidos graxos livres do óleo de linhaça ${ }^{16}$. $\mathrm{O}$ pH de ambas as nanocápsulas de PCL mostram valores bem semelhantes e levemente ácidos. A comparação das médias dos parâmetros estudados: tamanho, índice de polidispersão, potencial zeta e $\mathrm{pH}$, demonstra que não houve diferenças significativas entre as nanocápsulas contendo PCL e OL (ANOVA, 1 via, p>0,05). 
As nanocápsulas desenvolvidas com o polímero EUD RL100 não apresentaram diferença significativa entre si em todos os parâmetros avaliados (ANOVA, 1 via, $p>0,05$ ). Os resultados para o tamanho de gotícula demonstram que ambas apresentam valores satisfatórios, porém os valores foram menores nas formulações contendo metade da concentração de óleo $(1,5 \%)$. Essa observação pode ser explicada novamente pela alteração na viscosidade da fase orgânica que, por empregar uma menor quantidade do óleo, facilita a formação das partículas, apresentando diâmetros menores ${ }^{23,31}$. Nos resultados de índice de polidispersão observou-se que os valores da formulação NC-EUD-A foi mais elevado, indicando baixa homogeneidade do sistema. O polímero EUD RL100 apresenta características catiônicas decorrentes do grupo amônio quaternário presente em sua estrutura, o que justifica o potencial zeta positivo encontrado nas análises ${ }^{35}$. Quanto ao pH das suspensões de nanocápsulas, foram observados valores levemente ácidos.

Comparando as três formulações, não foram observadas diferenças significativas nos parâmetros de diâmetro médio e índice de polidispersão. No entanto, as avaliações de pH mostraram uma redução significativa nos valores quando o EUD RL100 foi empregado. Uma vez que a presença do polímero pode apresentar vantagens no desempenho da formulação, as partículas contendo polímeros podem ser consideradas promissoras para a veiculação do OL sendo um sistema de liberação promissor para futuros estudos, podendo ser incorporado em formulações para uso oral e então o OL adequadamente absorvido através trato gastrointestinal.

\section{Considerações Finais}

Este estudo demonstrou a viabilidade de preparação de nanoemulsões contendo óleo de linhaça em duas concentrações, bem como de suspensões nanocápsulas utilizando polímeros de diferentes naturezas (EUD RL100 e PCL). As formulações apresentaram características físico-químicas satisfatórias para sistemas coloidais. É importante ressaltar ainda, que as formulações com menor concentração de óleo de linhaça apresentaram tamanho e índice de polidispersão mais adequados. Desta forma, as formulações desenvolvidas são promissoras para a veiculação do óleo de linhaça para ampliar suas aplicações nutracêuticas e farmacológicas.

\section{Agradecimentos}

Os autores agradecem a Professora Dra. Cristiane de Bona da Silva pelo empréstimo de equipamento para as análises de tamanho de partícula/gotícula, IPD e potencial zeta.

\section{Referências}

1. Galvão EL, Silva DC, Silva JO, Moreira AVB, Sousa EMB. Evaluation of the antioxidant potential and sub-critical extraction of linseed oil. Food Sci. Technol. 2008; 28 (3): 551-557.

2. Touré $A$, Xueming $X$. Flaxseed: source, biosynthesis, metabolism, antioxidant, activity, bio-activity, components, and health benefits. CRFSFS. 2010; 9:261-269.

3. Andrade PMM, Carmo MGT. Ácidos graxos n-3: um link entre eicosanoides, inflamação e imunidade. INJC, UFRJ. 2006; 8(3):135-143.

4. Nykter M, Kymäläinen H, Gates F. Quality characteristics of edible linseed oil. Agr. Food Sci. Finland. 2006;15:402-413.

5. Martin CA, Almeida VV, Ruiz MR, Visentainer JEL, Matshushita M, Souza NE et al. Ácidos graxos poliinsaturados ômega 3 e ômega 6: importância e ocorrência em alimentos. Rev. Nutrição. 2006; 19(6): 761-770.

6. Martins MB, Suaiden AS, Piotto RF, Barbosa, M. Propriedades dos ácidos graxos poliinsaturados-Omega3 obtidos de óleo de peixe e óleo de linhaça. J. Health Sci. Inst 2008; 26(2): 153-56.

7. Silva FAM, Borges MFM, Ferreira MA. Métodos para avaliação do grau de oxidação lipídica e da capacidade antioxidante. Quím.Nova. 1999; 22(1):94-103.

8. ROSSI-BERGMANN, B. A nanotecnologia: da saúde para além do determinismo tecnológico. Cienc. Cult. 2008;60(2): 54-57.

9. SESSA, M. Nanoencapsulation of bioactive compounds for food applications. 2012. 178p. Tese (Doutorado) Universidade de Salermo, 2012.

10. Couvreur P, Vauthier C. Nanotechnology: intelligent design to treat complex disease. Pharm. Res. 2006; 23:1417-1450. 
11. Wu L, Zhang J, Watanabe W. Physical and chemical stability of drug nanoparticles.Adva. Drug Delivery Rev. 2011; 63:456-469.

12. Koroleva, MY, Yurtov, EV. Nanoemulsions: the properties, methods of preparation and promising applications. Russ. Chem. Rev. 2012; 81(1): 21-43.

13. Bromberg R. Aspectos relacionados ao uso da nanotecnologia na indústria de alimentos. TecnoCarnes Expresso. 2010;72-76.

14. Schaffazick SR, Guterres SS, Freitas LL, Pohlmann AR. Caracterização e estabilidade físico-química de sistemas poliméricos nanoparticulados. Quím. Nova. 2003:5:726-737.

15. Guterres SS, Schaffazick SR, Pohlmann AR. Preparação e aplicações de nanopartículas para liberação controlada de fármacos. In: Morales MM . Terapias Avançadas - células-tronco, terapia gênica e nanotecnologia aplicada à saúde. São Paulo: Atheneu. 2007; 247-264.

16. Mora-Huertas CE, Fessi H, Elaissari A. Polymer-based nanocapsules for drug delivery. Int. J. Pharm.2010;385:113-142. 17. Couvreur P, Barratt G, Fattal E, Legrand P, Vauthier C. Nanocapsule technology: a review. Crit. Rev. Therap. Drug Delivery Syst. 2002; 19:99-134.

18. Bouyer E, Mekhloufi GM, rosilio V, grossiord J, agnely F. Proteins, polysaccharides, and their complexes used as stabilizers of emulsions: Alternatives to synthetic surfactants in the pharmaceutical field? Int. J Pharm. 2012; 436:359 - 378. 19. Rieux A, Fievez V, Garinot M, Schneider YJ, Préat V. Nanoparticles as potential oral delivery systems of proteins and vaccines: a mechanistic approach. J.Control. Release. 2006;116:1-27.

20. Chassot JM, Ribas D, Silveira EF, Grunspan LD, Pires CC, Farago PV et al. Beclomethasone dipropionate-loaded polymericnanocapsules: development, in vitro cytotoxicity, and in vivo evaluation of acute lung injury. J. Nanosci. Nanotechnol. 2014; 15:855-864.

21. Santos SS, Lorenzoni A, Ferreira LM, Mattiazzi J, Adams Al, Denardi LB et al. Clotrimazole-loaded Eudragit $囚$ RS100 nanocapsules: Preparation, characterization and in vitro evaluation of antifungal activity against Candida species. Mater Sci Eng C. 2013; 33: 1389-1394.

22. Fessi H, Puisieux F, Devissaguet JP, Ammoury N, Benita S. Nanocapsule formation by Interfacial Polymer Deposition following solvent displacement. Int. J. Pharm. 1989; 55:1-4.

23. Bouchemal K, Briançon S, Perrier E, Fessi H. Nano-emulsion formulation using spontaneous emulsification: solvent, oil and surfactant optimisation. Int. J. Pharm. 2004; 280:241-251.

24. Santos SS, Lorenzoni A, Pegoraro NS, Denardi LB, Alves SH, Schaffazick SR et al. Formulation and in vitro evaluation of coconut oil-core cationic nanocapsules intended for vaginal delivery of clotrimazole. Colloids Surf. B Biointerfaces. 2014; 116: 270-276.

25. Ferreira LM, Cervi VF, Gehrcke M, Da Silveira EF, Azambuja JH, Braganhol E et al. Ketoprofen-loaded pomegranate seed oil nanoemulsion stabilized bypullulan: selective antiglioma formulation for intravenous administration. Colloids Surf. B Biointerfaces. 2015; 130:272-277.

26. Ferreira LM, Sari MHM, Cervi, VF, Gehrck M, Barbieri AV, Zborowski VA et al. Pomegranate seed oil nanoemulsions improve the photostability and in vivo antinociceptive effect of a non-steroidal antinflammatory drug. Colloids Surf. B Biointerfaces. 2016; 144: 214-221.

27. Camargo ZT, Caballero NED, Guterres SS. Nanocápsulas poliméricas deformáveis no encapsulamento de bioativos. PI 0905347, 15 dez. 2009, 07 jul. 2013.

28. Solans, C, Izquierdo, P, Nolla, J, Azemar, N, Garcia-Celma, MJ, Nano-emulsions. Curr. Opin. Colloid Interface Sci. 2005; 10: $102-110$.

29. Vyas TK, Shahiwala A\& Amiji MM. Improved oral bioavailability and brain transport of Saquinavir upon administration in novel nanoemulsion formulations. Int. J. Pharm. 2008; 347: 93-101.

30. Silveira EF, Chassot JM, Teixeira FC, Azambuja JH, Debom G, Beira FT et al. Ketoprofen-loaded polymeric nanocapsules selectively inhibit cancer cell growth in vitro and in preclinical model of glioblastoma multiforme. Investig. New Drugs. 2013; 31:1424-1435.

31. Sadurnì N, Solans C, Azemar N, Garcia-Celma MJ. Studies on the formation of O/W nano-emulsions, by low-energy emulsification methods, suitable for pharmaceutical applications. Eur. J. Pharm. Sci. 2005; 26:438-445.

32. Larini, L. Fármacos e medicamentos. Porto Alegre: Artmed; 2008. 
33. Almeida JS, Lima F, Da Ros S, Bulhões LOS, Carvalho LM, Beck RCR. Nanostructured Systems Containing Rutin: In Vitro Antioxidant Activity and Photostability Studies. Nanoscale Res Lett. 2010; 5:1603-1610.

34. Rigo LA, Silva CR, Oliveira SM, Cabreira TN, Da Silva, CB, Ferreira J et al. Nanoencapsulation of rice bran oil increases its protective effects against UVB-radiation induced skin injury in mice. Eur. J. Pharm. Biopharm. 2015; 93,11-17.

35. Wulff R, Leopold CS. Coatings from blends of Eudragit $\mathrm{RL}$ and $\mathrm{L} 55$ : A novel approach in pH-controlled drug release. Int. J. Pharm. 2014;476(1-2), 78-87.

\section{Cristina Rigon}

Endereço para correspondência - Universidade Federal de Santa Maria, Centro de Ciências da Saúde, Departamento de Farmácia Industrial - Prédio 26,

Bairro: Camobi, CEP: 97105900 , Santa Maria, RS, Brasil.

E-mail:cristinarigon@hotmail.com

Lattes: http://lattes.cnpq.br/0048655224986199

Laura Minussi Giuliani - laura.mgiuliani@gmail.com

Mônica Fabiele Lorensi Stangarlin - monica_stangarlin@yahoo.com.br

Juliane Mattiazzi - jujumattiazzi@yahoo.com.br

Felipe Pereira Gomes - felipe.pereiragomes@gmail.comi

Natháli Schopf Pegoraro - nathali.farmacia@hotmail.com

Letícia Cruz - leli.cs@gmail.com

Luana Mota Ferreira - luanamotaferreira@yahoo.com.br

Enviado em 22 de junho de 2016. Aceito em 17 de janeiro de 2017. 